\title{
XX.
}

\section{Über eine Degenerationscyste der Nebenniere mit kompensatorischer Hypertrophie.}

(Aus dem Patbologisch-anatomischen Institut der k. Universität zu Turin.) Von

\author{
Dr. G. Marchetti.
}

(Hierzu Taf. XVI.)

In nachstehenden Zeilen berichte ich über einen in der Pathologie der Nebennieren mehr ein Unikum als eine Seltenheit darstellenden Fall, der wegen der Folgen, zu denen er Anlaß gab, eine besondere Bedeutung erlangt und deshalb ein gewisses Interesse bieten dürfte.

Bei der Autopsie einer an eitriger Peritonitis gestorbenen 50 jährigen Frau wurde in der rechten Nebennierengegend ein großer, praller, elastischer, fluktuierender Tumor angetroffen. Derselbe drängte sich oben gegen die untere Leberwand, infolgedessen die Leber an dieser Stelle eine tiefe Höhlung anfwies; unten lag er dem oberen Pole der rechten Niere und deren Vorderfläche auf, wodurch die Niere etwas zusammengedrückt und verunstaltet wurde; hinten lag er den Lendenmuskeln an; vorn war er mit Peritonaeum bekleidet und bildete schlaffe Verwachsungen mit der unteren Hohlvene, in welche er eine nicht unbeträchtliche Vene sandte. Der Tumor wurde mit der Niere und einem ziemlichen Abschnitt der aufsteigenden Hohlvene abgetragen; er war oval gestaltet, mit zwei Vorwölbungen, einer binteren und einer vorderen, die durch eine Medianfurche getrennt waren, an welcher man einen harten, resistenten Strang tastete. Die hintere Fläche ließ bei aufmerksamer Betrachtung orangerote Flecken durchschimmern, und solche gewahrte man auch auf der vorderen Vorwölbung. Der Wand dieser letzteren, und genauer gesagt nahe der Hohlvene, war ein Strang aufgelagert, der sich durch sein Aussehen sogleich als Nebennierensubstanz za erkennen gab. Er erstreckte sich vom Gipfel der Geschwulst bis zu deren unterstem Abschnitt, ragte gegen den medianen Teil $1 \frac{1}{2} \mathrm{~cm}$ weit frei hervor und verdünnte sich all- 
mählich gegen die beiden Enden, wo er ins Innere der Geschwulst $\mathrm{zu}$ d̉ringen und sich $\mathrm{zu}$ verlieren schien. Er war $11 \mathrm{~cm}$ lang und etwa $1 \mathrm{~cm}$ dick (Taf. XVI, Fig. 1).

Bei einem gegen die Hälfte der Geschwulst gemachten Querdurcbschnitt trat eine hellgelbe, dicke, körnige Flüssigkeit heraus und die Geschwulst erschien von einem weiten Hohlraum gebildet, der durch ein vollständiges, mit dem dicken fibrösen Strang zwischen den beiden Vorwölbungen zusammenfallendes Septum in zwei Teile geteilt wurde. Die diese Höhlen umgrenzenden Wandungen hatten keine gleichmäßige, sondern eine zwischen 2 und $4 \mathrm{~mm}$ schwankende Dicke und wiesen hier und dort, genauer gesagt an den oben erwähnten gefleckten Stellen, gelbliche Streifen auf. Der an der vorderen Vorwölbung hervorragende Strang zeigte beim Durchschnitt die charakteristische Färbung der Nebennierenzonen; diese Substanz erstreckte sich auf einer Seite mehr als auf der anderen in die Dicke der Cystenwandung. Das die beiden Höhlen trennende Septum wies weitere kleine Cysten mit dem gleichen Inhalt und Inseln von orangegelber Substanz auf.

Die maximalen Durchmesser der Cyste maßen: der Jongitudinale $11 \mathrm{~cm}$, der von vorn nach hinten gerichtete $8,5 \mathrm{~cm}$, der transversale $7 \mathrm{~cm}$.

Die linke Nebenniere war deutlich hypertrophisch und wog $11,70 \mathrm{~g}$. Herr Prof. Foà forderte mich auf, diese pathologischen Stücke zu untersuchen.

Der Hauptzweck, den ich durch meine Untersuchungen zu erreichen suchte, war natürlich, die Natur und die Entstehungsweise der Cyste festzustellen; ich verließ mich dabei vollständig auf die histologische Untersuchung, da in der Krankheitsgeschichte alle Erscheinungen fehlten, die, wenn nicht die Natur, so doch das Bestehen der Geschwulst hätten vermuten lassen.

Als Fixierungsmittel benutzte ich Formalin für die Cyste, Sublimat-Müllersche Flüssigkeit für die linke Nebenniere. Die Cyste untersuchte ich an verschiedenen Stellen und zerschnitt besonders Stücke aus der Wandung, dem Septum und dem von der Nebenniere zurückgebliebenen Strang.

Die Cystenwand, die mit Hämatoxylin und Eosin gefärbt wurde, erscheint aus Bündeln derben Bindegewebes mit spärlichen

Virchows Archiv f. pathol. Anat. Bả. 172. Hft. 3. 
Kernen bestehend, an gewissen Stellen scheint sie sich in zwei Lamellen zu spalten: eine äußere und eine innere, zwischen denen sich Streifen von Epithelzellen der Nebennierenrindensubstanz finden. Diese Streifen sind diffus durch die ganze Cystenwand verbreitet und entsprechen jenen rotgelben Flecken, die bei der makroskopischen Untersuchung wahrgenommen wurden.

Die Schnitte, die dort angelegt wurden, wo der von der Nebenniere zurückgebliebene Strang sich mit der Cystenwand zu verschmelzen scheint, bieten ebenfalls einiges Interesse (Taf.XVI, Fig. 2). Das Nebennierenparenchym, das die normale Beschaffenheit verloren hat, besteht aus durch Bindegewebszüge voneinander getrennten Epithelinseln, die Bindegewebszüge vereinigen sich nach außen und nach innen und bilden zwei kompakte Lamellen, die die Kapsel umschließen und sich gegen die Cystenwand hin immer mehr einander nähern, wo sie sich dann vollständig vereinigen, jedoch lange Züge von Nebennierenzellen zwischen sich einschließend.

Der mittlere Abschnitt des besagten Stranges zeigt nämlich dort, wo er $1 \frac{1}{2} \mathrm{~cm}$ vorspringt, das gleiche Verhalten, d. h. die Nebennierensubstanz verliert sich hier zwischen den Bindegewebsbündeln der Cystenwand; während der frele Abschnitt gut erhalten, ja hypertrophisch ist.

Hier bestehen alle drei Zonen der Rindensubstanz, und die Hypertrophie wird besonders in der Zona fasciculata wahrgenommen. Näher der Cystenwand bemerkt man, daß die Inseln von Substantia fasciculata sich im Centrum vakuolisieren und die Elemente sich kreisförmig anordnen, als umgrenzten sie ein Harnkanälchen; sie platten sich $a b$, verflachen sich, sodaß dort, wo der Prozeß am meisten vorgeschritten ist, nur die Kerne den Bindegewebsbälkchen aufliegen. Mitunter zerstückeln sich diese Bälkchen, und dann fließen die Vakuolen ineinander und bilden kleine Cysten mit granulösem Inhalt.

Diese Erscheinung tritt viel deutlicher in den Schnitten des fibrösen Stranges auf, wo man auch makroskopisch kleine Cysten wahrnimmt. Unter dem Mikroskop erscheint er zum großen Teile aus derbem Bindegewebe bestehend, in dessen Dicke die Dilatation der Kapillaren, häufige Ablagerung von Kalksalzen 
and das Bestehen eines kleinen Angioms wahrgenommen werden. Außerdem gewahrt man Züge von Nebennierenzellen, die nicht unregelmäßig zerstreut, sondern meistens einer in große Inseln der gleichen Substanz auslaufenden Linie entlang angeordnet sind. Diese Substanz ist an gewissen Stellen sehr gut erhalten, an anderen weist sie Vakuolisation und die oben beschriebenen Cystenbildungen (Taf. XVI, Fig. 3) auf. Diese Hohlräume, die bisweilen direkt von Nebennierensubstanz umgrenzt sind, an anderen Stellen schon eine bindegewebige Verdickung aufweisen, haben vollkommen den gleichen Inhalt, wie die größeren Cysten, der unter dem Mikroskop sich als aus einer körnigen Masse bestehend erweist; weder gestaltete Elemente, noch Krystalle kommen darin vor, es scheint sich ausschließlich um Zellentrümmer zu handeln.

In der linken Nebenniere beobachtet man eine imposante Hypertrophie der ganzen Rindenzone, besonders der Zona fasciculata, die sehr ausgedehnt und mit dichtgedrängten Säulen versehen ist. Das Organ ist gänzlich kongestioniert, besonders auf der Grenze zwischen Zona fasciculata und Znna reticularis. Karyokinetische Figuren konnte ich trotz aufmerksamster Nachforschung nicht auffinden. Dagegen nahm ich eine andere charakteristische Erscheinung wahr: in den mehr gegen die Peripherie gelegenen Fascicularparenchyminseln bestehen kreisförmige Räume, die, wie es scheint, im Centrum derselben sich zu bilden beginnen und immer mehr zunehmen, indem sie die Nierenelemente verrïcken, die sich ringsherum anordnen, und die oft ein den gewundenen Nierenkanälchen ähnliches Aussehen erlangen. Das Lumen derselben ist von einigen abgeschuppten Zellen und stets von einer körnigen Substanz eingenommen (Taf. XVI, Fig. 4). In diesen Räumen habe ich nie Blutelemente angetroffen, dagegen bemerkte ich die Anwesenheit von Nebennierenzellen im Inhalt der centralen Gefäße des Organs. Die granulöse Substanz der neugebildeten Höhlen scheint von den umliegenden Zellen herzurühren, die an ihren Rändern bisweilen kleine, mit Eosin sich rosa färbende Kügelchen aufweisen; es sind dies ganz ähnliche Kügelchen wie sie $\mathrm{F}_{0}$ à bei den gewöhnlichen Versuchstieren nach Injektionen von toxischen Substanzen angetroffen hat.

Aus der histologischen Untersuchung geht hervor, dab wir uns vor einem Vorgang ziemlich alten Datums befinden, und es 
wäre uns schwer gefallen, die Entstehung dieser Cyste zu erklären; wenn wir nicht die kleinen recenten Cysten angetroffen hätten.

Da eine ähnliche pathologische Erscheinung bisher noch nicht beschrieben worden ist, vermute ich, daß es sich entweder um einen embryogenen Vorgang handelte, oder um eine durch Erweichung bedingte Pseudocyste in einer in der Nebenniere entstandenen Geschwulst, wie solche von Thorel beschrieben worden sind, oder auch um eine Blutcyste als Folge einer Nebennierenhämorrhagie.

Ich habe nichts angetroffen was die Annahme eines embryonalen Einschlusses oder des vorherigen Bestehens eines Neoplasmas rechtfertigen könnte.

In der Literatur finden sich einige Fälle von Blatcysten beschrieben, die mit dem vorliegenden eine gewisse Ähnlichkeit zu haben scheinen. Bayer, Chiari, Routier und Leconte beschrieben Nebennierentumoren, die aus großen Blutcysten bestanden und die klinische Symptome, wie lokalen Schmerz und Erbrechen, gegeben hatten; die Cysten nahmen die ganze Nebenniere ein, deren Substanz entweder gänzlich zerstört war oder eine schwere fettige Degeneration erfahren hatte. Keiner von diesen Autoren bemerkte eine Modilikation in der betreffenden Nebenniere.

Neuerdings hat Simmonds einige Fälle von Nebennierenhämorrhagie gesammelt, in denen diese durch embolische Thrombose der Gefäße des Organs bedingt war. Meistens waren es circumscripte oder pünktchenförmige Hümorrhagien, sehr selten war die Hämorrhagie eine derartige, daß sie zu Blutcysten Veranlassung gab. In dem intakt gebliebenen'Teile wurde eine vicariierende Hypertrophie angetroffen.

Da jedes von den von Arnaud als pathognomonisch für Nebennierenhämorrhagie angegebenen Symptomen fehlt, da die zurückgebliebene Nebennierensubstanz, statt zu Grunde zu gehen oder zu degenerieren, gut erhalten und an gewissen Stellen hypertrophisch ist, wegen des Inhalts der Cyste und endlich wegen der in der Nebenniere der anderen Seite angetroffenen Hypertrophie, bin ich zur Überzeugung gelangt, da 3 der Entstehungsprozeß der Cyste ein ganz anderer gewesen sein und viel langsamer stattgefunden haben mußte, als wenn es sich um Nebennierenhämorrhagie gehandelt hätte. 
Auf Grund der histologischen Untersuchung komme ich dagegen zum Schlusse, daß die Cyste durch abnorme Sekretion mit Zerfall der Zellenelemente entstanden ist, und zwar aus folgenden Gründen:

1. wegen der abnormen Anordnung der verschiedenen Zonen;

2. wegen der Bildung der Hohlräume und Kanälchen innerhalb der Inseln und der Zellenbündel;

3. weil sich in diesen Räumen eine von den umliegenden Zellen herrührende granulöse Substanz angesammelt hat;

4. weil sich die Elemente gegen die Bindegewebsbälkchen abgeplattet haben und letztere, dadurch daß sie sich verdünnten und zerstückelten, immer größere Höhlen entstehen ließen.

Mancher könnte einwenden, daß es zur Entstehung einer so voluminösen Cyste einer mechanischen Ursache bedurfte und daß sich andrerseits nicht von Retentionscysten sprechen läßt, da das Bestehen eines Ausführungsganges nicht nachgewiesen ist. Einerseits weiß man, daß auch bei Drüsen mit deutlich nachgewiesenem Ausführungsgang, die Obliteration desselben nicht zur Bildung von Cysten führt und daß die experimentelle Ligatur des Ganges nur Retrodilatation der Drüsenkanäle und -Blindsäcke hervorruft. Andrerseits läBt sich die Cystenbildung in der Leber und der Niere nicht auf den Retrodilatationsmechanismus zurückführen. Cornil und Ranvier bemerken, daß „les kistes dits de rétention ne reconnaissent pas uniquement pour cause l'accumulation du produit de sécrétion consécutif à l'obliteration d'un conduit glandulaire".

In unserem Falle ist die Cyste wirklich durch eine Regressivveränderung der Zellen entstanden. Deshalb läßt sich annehmen, daß zu Anfang die Verschmelzung der neugebildeten Hohlräume infolge der durch die abnorme Sekretionsfunktion bedingten Druckveränderung stattgefunden und die Ansammlung der Flüssigkeit in ihnen den neuen Räumen eine regelrecht sphärische Gestalt gegeben habe. Diese primären Cystenbildungen sind an verschiedenen Stellen der Nebenniere zustande gekommen; in der Folge haben sich andere neugebildete kleine Cysten mit den größeren verschmolzen, die dann infolge des Zerfalls des sie trennenden Septums ebenfalls ineinanderflossen. 
Eine so tiefe Veränderung rief in dem vom Organ übrig gebliebenen Teil eine vicarierende Hypertrophie hervor, in gleicher Weise wie bei gewissen Organen, z. B. bei den Nieren, wenn ein pathologischer Vorgang einen Teil des Organs der Funktion entzieht, der übrige intakte Teil der Hypertrophie verfällt.

Diese Kompensation ist bei der Nebenniere der anderen Seite eine imposante und dies bestätigt in der Pathologie des Menschen, was Stilling bei seinen Experimenten beobachtete. Stilling erhielt experimentell eine kompensatorische Hypertrophie, aber nur bei jungen Tieren, bei denen der Prozeß rasch seinen Anfang nimmt, nämlich zwei oder drei Tage nach erfolgter Exstirpation. Bei ausgewachsenen Tieren dagegen wird auch drei Monate nach der Operation keine merkliche Strukturveränderung wahrgenommen. Die Hypertrophie hatte in seinen Fällen hauptsächlich in der Substantia fasciculata ihren Sitz. Auch Sim monds erhielt ähnliche Resultate und versichert, daß solche Hypertrophie nur bei jungen Individuen stattfindet; ja damit erklärt er die Seltenheit des Kompensationsprozesses beim Menschen, denn da er im Jugendalter stattfindet, hört er mit dem weiteren Fortschreiten des Alters auf.

Vergleichen wir unsern Fall mit den von diesen beiden Forschern erhaltenen Tatsachen, so können wir mit ihnen schließen, da $\beta$ die Nebennieren eine vicarierende Funktion haben; doch müssen wir bemerken, daß diese Funktion sich nicht nur im Jugendalter entfaltet. Denn wenn wir auch die Schlüsse Si m m onds gelten lassen und annehmen wollten, daß in unserem Falle die rechte Nebenniere in den ersten Lebensjahren zu funktionieren aufgehört habe, so bleibt doch immer die Tatsache, daß die kompensatorische Hypertrophie in einem Alter von 52 Jahren angetroffen wurde. Andrerseits ist der Prozeb, der nach Exstirpation auftritt, wo die Funktion ganz plötzlich einem einzigen Organ aufgebürdet wird, ganz verschieden von dem, der stattfindet, wenn die Zerstörung langsam und allmählich erfolgt.

Die von mir gemachte Beobachtung des Bestehens von Räumen im Innern der Bündel der hypertrophischen Substanz bestätigte mir, da $\beta$ auch in der linken Nebenniere eine abnorme Sekretion oder eine übermäßige Funktionstätigkeit stattgefunden 
habe. Um festzustellen, ob und in welchen Fällen etwas Ähnliches vorkomme, unternahm ich eine Reihe Untersuchungen.

Von etwa 700 Autopsien, die ich im Laufe des Jahres in dem hiesigen Institut ausführte, habe ich 42 Nebennieren gesammelt, die makroskopisch Merkmale von einigem Interesse aufwiesen; vergleichshalber führte ich noch andere Untersuchungen an normalen und gut erhaltenen Nebennieren aus.

$\nabla_{0}$ allen diesen menschlichen Nebennieren, die verschieden alten und an verschiedenen Krankheiten gestorbenen Individuen angehörten, gab nur eine einzige einen ähnlichen histologischen Befund, und diese stammte von einer Wöchnerin.

Ich weiß nicht, inwiefern das Puerperium sich mit dem von mir erhobenen Befund in Beziehung bringen lasse, denn aus einem einzigen Befund läßt sich kein sicherer Schluß ziehen. Vielleicht ist die Annahme statthaft, daß die Schwangerschaft zuweilen von einer übermäßigen Funktionstätigkeit der Nebennieren begleitet werde.

Meinen Lebrer, Herrn Prof. Foà sage ich hier meinen besten Dank dafür, daß er mich einen so interessanten Fall hat untersuchen lassen.

\section{Erklärung der Abbildungen auf Tafel XVI.}

Fig. 1. Makroskopisches und topographisches Aussehen des pathologischen Stückes.

1 Cyste, 2 Rest von der rechten Nebenniere, 3 aufsteigende Hoblvene, 4 Niere.

Fig. 2. Übergangsstelle vom Nierenparenchym zur Cystenwand.

Fig. 3. Schnitt durch das Septum zwischen den beiden Cystenvorwölbungen.

a Innenwand, a' Außenwand, b recente Cysten, b' ältere Cysten, c Angiom.

Fig. 4. Hypertrophiscbe linke Nebenniere.

aa neugebildete Höhlen in der Substantia fasciculata, b $\mathbf{A b}$ schuppungszellen.

\section{Literatur.}

Bayer: Journal l'Experience 10 no. 1817.

Tizzoni: Sur la physiopathologie des C. S. Archives it. de Biol. 1884.

Chiari: Wiener medizinische Zeitung 1885.

Mattei: Recherches sur l'an. path. des C. S. 1865 . S. 386. 
Routier: Société de Biologie. Paris 1894.

Leconte: Thèse de Paris 1897. Etudes sur les hémorragies des C. S.

F. Arnaud: Les hémorrhagies des C. S. Arch. gén. de Médecine. 1900. IIe ann.

Foà, P.: Contribuzione anatomica sperimentale alla fisiologia patologica delle C. S, 1901.

Stilling: Revue de Médecine 1888. S. 459.

Simmonds: Über kompensatorische Hypertrophie der Nebenniere. Dieses Archiv Bd. 153, 1898.

Derselbe: Über Nebennierenblutungen. Dieses Archir 1902.

Thorel, Ch.: Zur Frage der Erweichungscysten in Geschwülstën der Nebenniere 1902.

Cornil et Ranvier: Manuel d'Histologie path. 1902.

\title{
XXI.
}

Über Nebennierenschrumpfung bei Morbus Addison.

\author{
Von \\ Dr. M. Simmonds, \\ Prosektor am Allgem. Kraukenhause Hamburg-St. Georg.
}

Atrophische Zustände der Nebennieren sind kein übermäßig seltenes Vorkommnis. Sehe ich von der angeborenen Aplasie des Organpaars bei Anencephalen ab und von der kongenitalen Hypoplasie der einen Nebenniere, die gelegentlich bei sonst normalen Individuen als zufälliger Nebenbefund anzutreffen ist, so bleiben diejenigen Formen übrig, welche an schwere Cirkulationsstörungen oder an tuberkulöse Erkrankungen des Organs sich anschließen oder endlich idiopathisch auftreten. Bei weitem am häufigsten sind unter diesen Formen der Nebennierenschrumpfung, die durch Tuberkulose hervorgerufenen. Der Prozeß kann dabei, wie ich das früher ${ }^{1}$ ) gezeigt habe, so gründich und mit fast vollkommener Verödung des Parenchyms ausheilen, daß es bisweilen schwer hält, das Grundleiden ausfindig

1) Über kompensatorische Hypertrophie der Nebennieren. Dies. Archiv Ad. 153 , S. 143 . 

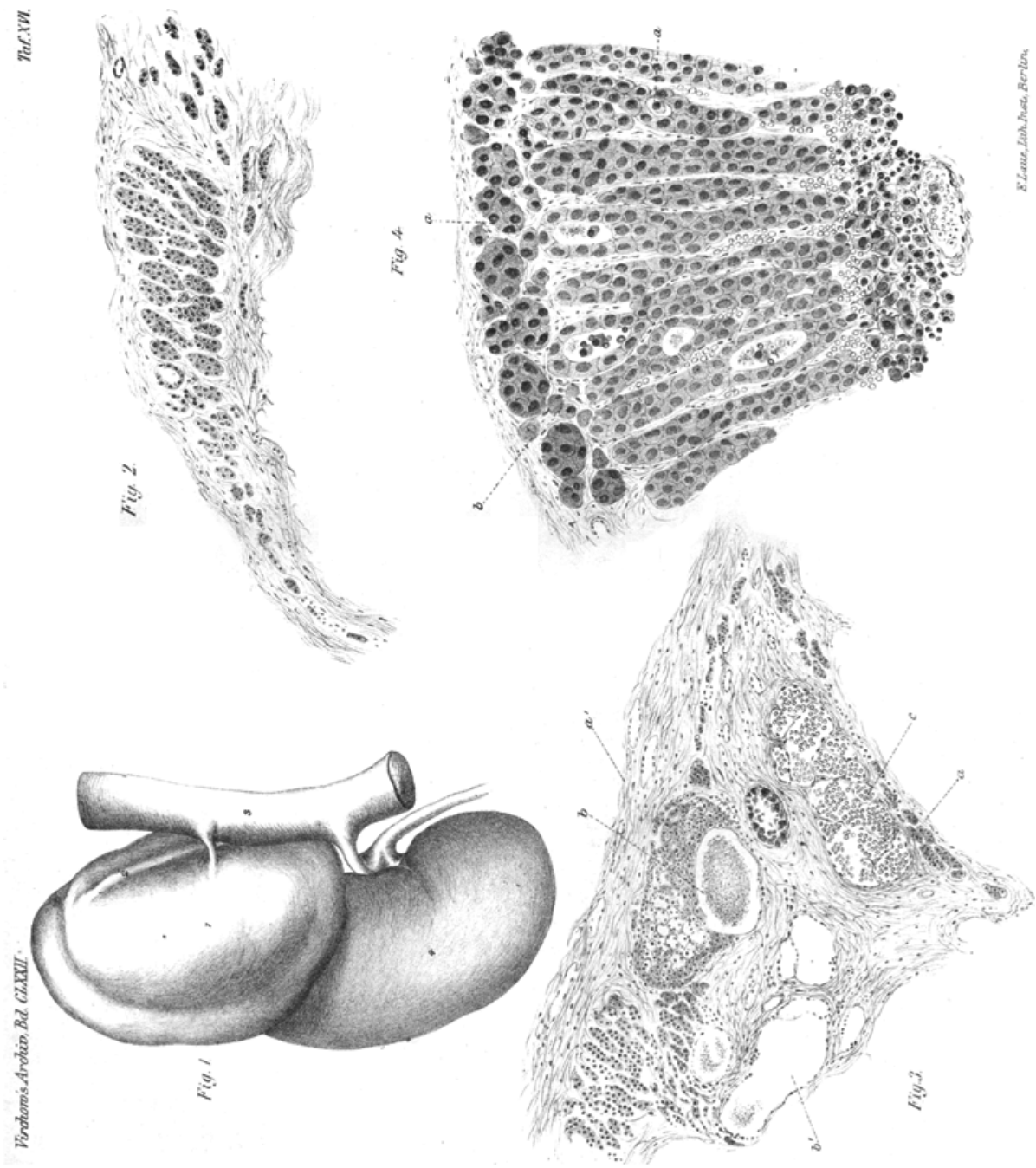